\title{
Analysis of the Lipid Metabolism and Related Factors Among Male Employees in Guangzhou From 2017 to 2019
}

wenting wei ( $\nabla$ dr_wenting@yeah.net )

Guangdong Academy of Medical sciences

yeran jia

Guangdong Academy of Medical Sciences

Wenfeng zhan

Guangdong Academy Of Medical Sciences

Faping Li

Guangdong Academy Of Medical Sciences

Pingyou Zhang

Guangdong Academy Of Medical Sciences

Hongxuan Zhang

Guangdong Academy Of Medical Sciences

Research

Keywords: community residents, dyslipidemia, prevalence

Posted Date: September 22nd, 2020

DOl: https://doi.org/10.21203/rs.3.rs-78254/v1

License: (9) (i) This work is licensed under a Creative Commons Attribution 4.0 International License.

Read Full License 


\section{Analysis of the lipid metabolism and related factors among male 2 employees in Guangzhou from 2017 to 2019}

\section{Abstract}

Background: To analyze abnormal plasma lipids during physical examination of adult men in a community hospital and to provide the basis for prevention and control measures.

Methods: Fasting serum lipids (TG, TC, LDL-C, HDL-C) were measured in 439 adult men in a community hospital from 2017 to 2019 . The results were analyzed by age.

Results: The prevalence of dyslipidemia was $15.72 \%, 19.36 \%$, and $20.50 \%$ in 2017,2018 , and 2019 , respectively. The abnormal rate of HDL-C increased significantly-from $2.96 \%$ in 2017 to $9.11 \%$ in 2018; it remained at $8.88 \%$ in 2019. The abnormal rate of TG and HDL-C increased gradually from age 50 years, and the highest was in those aged 60-65 years. The study also found that TC and LDL-C index levels peaked in the 55-65-year-old group; these levels then decreased with age. TG and LDL-C increased with age while HDL-C decreased with age. The increase in plasma lipid levels and obesity in young people was significantly higher than those in middle-aged people.

Conclusion: The rate of dyslipidemia among adult men in Guangzhou increases over time and with age. The prevention and treatment of dyslipidemia (awareness, treatment, and control) should be urgently strengthened to reduce the incidence of cardiovascular and cerebrovascular diseases and improve the level of community health services.

Key words: community residents; dyslipidemia; prevalence

\section{Introduction}

Guangzhou is an economically developed city in southern China. It is a large city with major industries and a large service sector. It has a huge population including both a local population and a transient population. Public health is an important issue in all mega-cities. Infectious diseases such as the novel 
coronavirus pneumonia require great attention. However, non-communicable diseases are the cornerstone of public health. With changes in residents' lifestyle, chronic non-communicable diseases including cardiovascular and cerebrovascular diseases, diabetes, and other diseases have become an important factor affecting the health of urban residents. ${ }^{[1][2]}$

Studies have shown that the prevalence of dyslipidemia in Chinese adults is gradually rising. ${ }^{[3]}$ Dyslipidemia has become the main risk factor for cardiovascular and cerebrovascular diseases in adults, and its main harm is to increase the risk of atherosclerosis causing coronary heart disease, stroke, and other diseases. ${ }^{[4]}$ Effective control of dyslipidemia is important in the prevention and control of atherosclerotic cardiovascular diseases. This study investigated and analyzed plasma lipids and related chronic diseases in Guangzhou from 2017 to 2019. We aimed to provide a basis for dyslipidemia prevention and control measures among community residents. The hospital physical examination center used here is the designated hospital for this cohort. Thus, we used convenience sampling to collect health examination data from this population.

\section{Objects and Methods}

General Subjects Information. We enrolled 439 adult men ups who underwent health check-ups over three consecutive years from 2017 to 2019. They were aged 25-65 years with an average of (49.04 \pm 10.46$)$ years including 27 aged 25-34 years, 98 aged 35-44 years, 147 aged 45-54 years, and 144 aged 55-65 years.

Collection of population medical examination data. Health examination data for these three years (2017, 2018, and 2019) were retrospectively analyzed including: (1) general conditions (gender, age); (2) medical history (hypertension, diabetes); (3) main measurement indexes (height, weight, waist circumference, plasma pressure, etc.) (4) laboratory test results (fasting plasma glucose (FPG), total cholesterol (TC), triglyceride (TG), high-density lipoprotein cholesterol (HDL-C), low density lipoprotein cholesterol (LDL-C), etc.) The study was approved by the hospital's ethics committee. The confidentiality and non-disclosure of patient information were confirmed during collecting and sorting data.

Execution methods of various physical examination contents. The "Guidelines for the Prevention and Treatment of Dyslipidemia in Chinese Adults (2016 Revision)" lists criteria according to Chinese 
ASCVD Level I Prevention Population Appropriate Levels of Plasma Lipids and Abnormal Stratification Criteria: $\mathrm{TG} \geq 2.26 \mathrm{mmol} / \mathrm{L} ; \mathrm{TC} \geq 6.22 \mathrm{mmol} / \mathrm{L}$ is hypercholesterolemia.HDL-C $<1.04$ $\mathrm{mmol} / \mathrm{L}$ is hypoHDL plasma disease. $\mathrm{LDL}-\mathrm{C} \geq 4.14 \mathrm{mmol} / \mathrm{L}$ is hypercholesterolemia, and dyslipidemia is one of the above conditions.

Statistical analysis. Data were derived from the medical record. SPSS software performed statistical analysis. The data were expressed by $\mathrm{x} \pm \mathrm{s}$, and the number of cases (\%) of counting data were expressed. The heatmap analysis uses ggplot2 software package in $\mathrm{R}$ language.

\section{Results}

\section{Profile changes of chronic diseases and plasma lipids in each subgroup from 2017 to $2019 . \quad$ In} general, 439 adult male health checkups were performed: smoking was seen in 213 subjects (48.52\%), hypertension in $72(16.40 \%)$, coronary heart disease in $90(20.50 \%)$, and diabetes in $46(10.48 \%)$; the obesity rates were 113 (25.74\%), 116 (26.42\%), and 102 (23.23\%) in 2017, 2018, and 2019, respectively. The basic information of each age group is shown in Table 1. We analyzed the metabolic indexes of all patients as well as the chronic disease complications. The heatmap shows the change trend of each observation index for patients at different times. According to the heatmap, plasma lipids of patients aged 25-34 is higher than that of patients aged 55-65. The heatmap data are shown in Figure. 1. We also made a subgroup analysis according to the combination of various diseases, and found that age is a change factor independent of chronic diseases during the time period of lipid metabolism disorder.

Changes in plasma lipids in each subgroup from 2017 to 2019. The average TG of plasma lipid level distribution in 2017,2018 , and 2019 is $(1.59 \pm 0.37) \mathrm{mmol} / \mathrm{L},(1.64 \pm 0.36) \mathrm{mmol} / \mathrm{L},(1.70 \pm 0.38) \mathrm{mmol} / \mathrm{L}$, respectively. The mean TC was $(5.46 \pm 0.59) \mathrm{mmol} / \mathrm{L},(5.48 \pm 0.58) \mathrm{mmol} / \mathrm{L}$, and $(5.51 \pm 0.53) \mathrm{mmol} / \mathrm{L}$, respectively. The average HDL-C is $(1.56 \pm 0.27) \mathrm{mmol} / \mathrm{L},(1.47 \pm 0.27) \mathrm{mmol} / \mathrm{L}$, and $(1.40 \pm 0.24)$ $\mathrm{mmol} / \mathrm{L}$, respectively. The mean LDL-C is $(2.46 \pm 0.44) \mathrm{mmol} / \mathrm{L},(2.52 \pm 0.42) \mathrm{mmol} / \mathrm{L}$, and $(2.61 \pm 0.53)$ mmol/L, respectively. TG and LDL-C gradually increase with age while HDL-C gradually decreases. Table 2 compares plasma lipid levels between age groups. The prevalence of dyslipidemia is 69 (15.72\%), 85 (19.36\%), and $90 \square 20.50 \% \square$ in 2017, 2018, and 2019, respectively. TG was seen in $51 \square 11.62 \%, 69 \square 15.72 \% \square$ and $68 \square 15.49 \% \square$ subjects in 2017,2018 , and 2019 , respectively. The rate of abnormal HDL-C increased significantly from $2.96 \%$ in 2017 to $9.11 \%$ in 2018 ; it was $8.88 \%$ in 2019 . The abnormal rates of TG and HDL-C gradually increase from age 45 with the highest rate in the 55-65 age group. The study also found that the TC and LDL-C index levels peaked in the 55-65 age group; 
thereafter, the TC and LDL-C index levels decreased with increasing age. The dyslipidemia rate and annual dyslipidemia rate were compared between different age groups in Tables 3 and 4.

\section{Discussion}

Dyslipidemia refers to the abnormal metabolism of lipids specifically those that decrease HDL-C levels and increase TG, TC, LDL-L, and LP-a. ${ }^{[5][6]}$ Clinical research results show that dyslipidemia is closely related to the occurrence of coronary heart disease and directly affects the prognosis of patients with coronary heart disease. ${ }^{[7][8]}$ In Hyperlipidemia can also lead to diabetes, impaired glucose tolerance, and high blood pressure. ${ }^{[9]}$ Incorporating plasma lipid testing into a health checkup project is essential to prevent atherosclerosis and dyslipidemia, promote a healthy lifestyle, guide a balanced diet, and prevent cardiovascular and cerebrovascular diseases. ${ }^{[10]}$

In China, people's living standards, work, and diet have recently undergone great changes. The lack of proper exercise and a rich diet has increased the incidence of dyslipidemia. ${ }^{[11]}$ Dyslipidemia is often accompanied by a variety of cardiovascular risk factors, which impacts governments, insurance companies, and the general population. ${ }^{[12][13]}$ Abnormal plasma lipid metabolism has become a public health problem and cannot be ignored by anyone. ${ }^{[14][15][16]}$

There are many studies on the metabolism of plasma lipids, but there are few studies on plasma lipids in the same population longitudinally. Here, 439 adult males were followed for three consecutive years (2017 to 2019). They were 25-65 years old, and 79 of them were 60 to 65 years. This paper does not include women because there are few women in the group; thus, only men were studied to eliminate the statistical differences brought by gender differences. The subjects mostly worked indoors.

The results of physical examination over three consecutive years showed that low density lipoprotein (LDL) increased with age while high density lipoprotein (HDL) decreased with age. The plasma lipids of the 25-34-year-old group was higher than those aged 55-65 years; the LDL increased, and the HDL decreased. There is no significant change in plasma lipid in the past three years in the 55-65 group. This may be because they are older and place more emphasis on diet and exercise. They also might have more risk factors for cardiovascular and cerebrovascular diseases. This population is more likely to take drugs to control plasma lipid metabolism. The changes in body weight are quite different between groups: The 29-34-year-old population had a particularly significant weight gain - the obesity rate increased from $8 \%$ to $12 \%$. The weight of the $55-65$ year old population is relatively stable with 
115 little change. In conclusion, longitudinal health data is important. This retrospective analysis suggests

116 that dyslipidemia is of concern among men in Guangzhou and is increasingly common.

118 Declarations

119 Ethical Approval and Consent to participate: not applicable.

122 Availability of data and material: not applicable.

123

124 Author's contribution: Wei Wenting, Jia Yeran, and Zhan Wenfeng performed data collation, form 125 analysis, survey, manuscript writing, review writing, and editing. Wei Wenting, Li Faping, and Zhang 126 Pingyou wrote the original draft. Zhang Hongxuan offered project management, supervision, writing 127 review, and editing. All authors read and approved the final version of the manuscript.

129 Acknowledgements: not applicable.

130

131 Funding: This research received no external funding.

132

133 Conflicts of Interest: The authors declare no conflict of interest.

134

135

136 


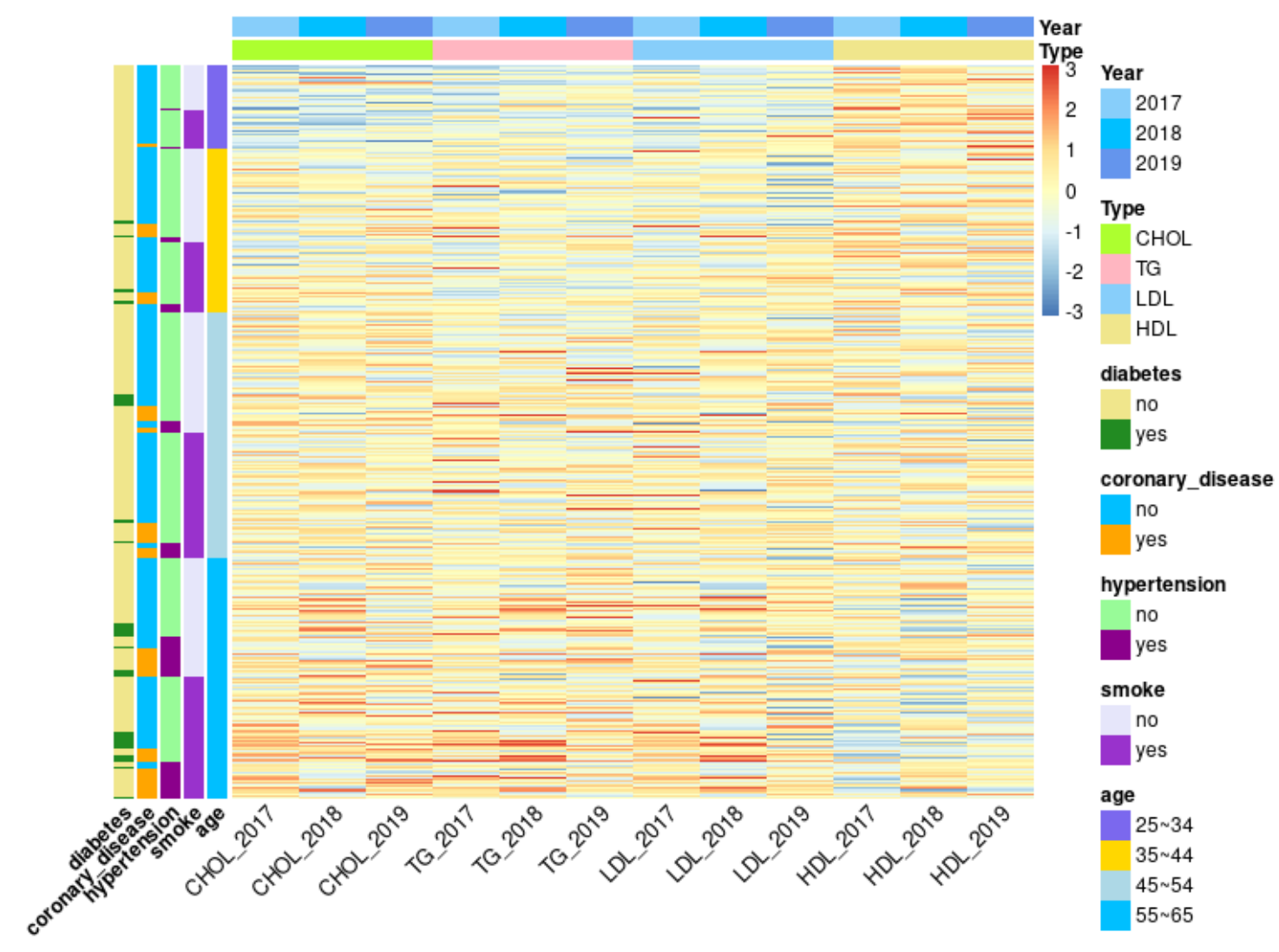


Figure 1. Analysis heatmap of risk factors related to plasma lipids (2017-2019).

Table 1. Basic Situation of Various Age Groups from 2017 to 2019.

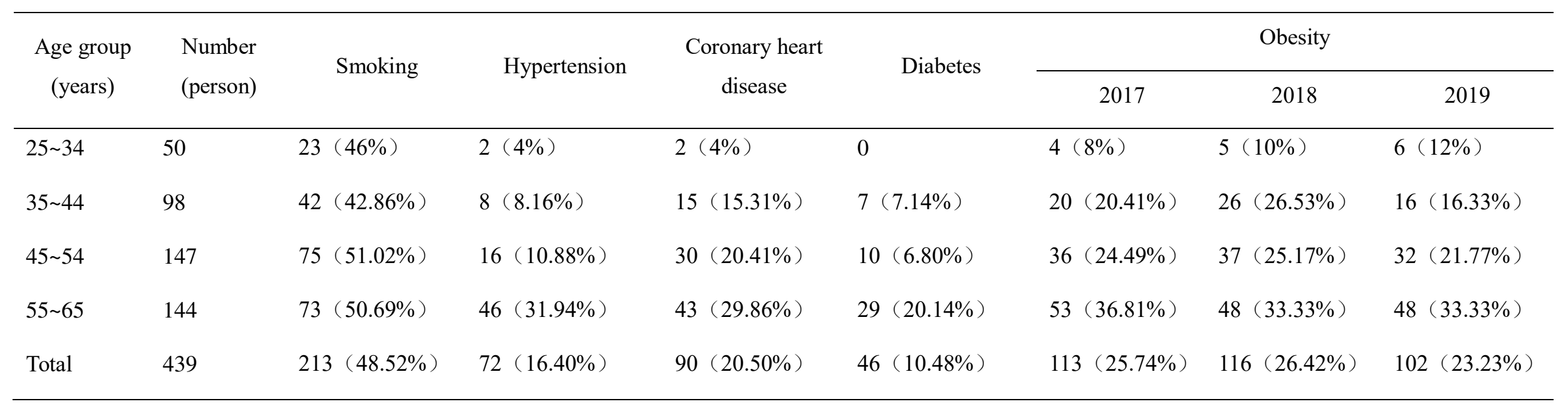

Table 2. Comparison of Plasma Lipid Levels in Different Age Groups from 2017 to 2019 (mmol/L).

\begin{tabular}{ccccccccccccc}
\hline $\begin{array}{c}\text { Age group } \\
\text { (years) }\end{array}$ & \multicolumn{3}{c}{ TC } & \multicolumn{3}{c}{ TG } & \multicolumn{3}{c}{ LDL-C } & \multicolumn{3}{c}{ HDL-C } \\
\cline { 2 - 12 } & 2017 & 2018 & 2019 & 2017 & 2018 & 2019 & 2017 & 2018 & 2019 & 2017 & 2018 & 2019 \\
\hline $25 \sim 34$ & $4.92 \pm 0.56$ & $4.99 \pm 0.55$ & $5.12 \pm 0.51$ & $1.37 \pm 0.25$ & $1.48 \pm 0.23$ & $1.54 \pm 0.21$ & $2.24 \pm 0.5$ & $2.31 \pm 0.25$ & $2.48 \pm 0.51$ & $1.72 \pm 0.33$ & $1.64 \pm 0.24$ & $1.57 \pm 0.33$
\end{tabular}




\begin{tabular}{|c|c|c|c|c|c|c|c|c|c|c|c|c|}
\hline $35 \sim 44$ & $5.36 \pm 0.57$ & $5.4 \pm 0.46$ & $5.50 \pm 0.46$ & $1.53 \pm 0.36$ & $1.57 \pm 0.24$ & $1.59 \pm 0.25$ & $2.4 \pm 0.45$ & $2.5 \pm 0.41$ & $2.52 \pm 0.55$ & $1.62 \pm 0.3$ & $1.5 \pm 0.31$ & $1.5 \pm 0.31$ \\
\hline $5 \sim 54$ & $5.51 \pm 0.53$ & $5.57 \pm 0.47$ & $5.55 \pm 0.48$ & $1.63 \pm 0.4$ & $1.64 \pm 0.37$ & $1.75 \pm 0.5$ & $2.5 \pm 0.43$ & $2.54 \pm 0.37$ & $7 \pm 0$ & $4 \pm 0.3$ & $.46 \pm 0.31$ & $.44 \pm 0.29$ \\
\hline $55 \sim 65$ & $5.66 \pm 0.55$ & $.61 \pm 0.65$ & $5.62 \pm 0.56$ & $1.66 \pm 0.35$ & $1.74 \pm 0.43$ & $1.77 \pm 0.35$ & $2.53 \pm 0.4$ & $2.59 \pm 0.49$ & $2.67 \pm 0.57$ & $1.48 \pm 0.31$ & $1.42 \pm 0.37$ & $1.4 \pm 0.26$ \\
\hline otal & $5.46 \pm 0.59$ & $5.48 \pm 0.58$ & $5.51 \pm 0.53$ & $1.59 \pm 0.37$ & $1.64 \pm 0.36$ & $1.70 \pm 0.38$ & $2.46 \pm 0.44$ & $2.52 \pm 0.42$ & $2.61 \pm 0.53$ & $1.56 \pm 0.32$ & $1.48 \pm 0.33$ & $1.46 \pm 0.29$ \\
\hline
\end{tabular}

Table 3. Comparison of Annual Dyslipidemia Rates from 2017 to 2019 (\%).

\begin{tabular}{|c|c|c|c|c|c|c|c|c|c|c|c|c|}
\hline \multirow{2}{*}{$\begin{array}{c}\text { Year } \\
\text { Inspection item }\end{array}$} & \multicolumn{4}{|c|}{2017} & \multicolumn{4}{|c|}{2018} & \multicolumn{4}{|c|}{2019} \\
\hline & $\mathrm{TC}$ & TG & LDL-C & HDL-C & $\mathrm{TC}$ & TG & LDL-C & HDL-C & $\mathrm{TC}$ & $\mathrm{TG}$ & LDL-C & HDL-C \\
\hline number & 42 & 51 & 5 & 13 & 46 & 69 & 4 & 40 & 52 & 68 & 1 & 39 \\
\hline Percentage & $9.57 \%$ & $11.62 \%$ & $1.14 \%$ & $2.96 \%$ & $10.48 \%$ & $15.72 \%$ & $0.91 \%$ & $9.11 \%$ & $11.85 \%$ & $15.49 \%$ & $0.23 \%$ & $8.88 \%$ \\
\hline Total & \multicolumn{4}{|c|}{$69(15.72 \%)$} & \multicolumn{4}{|c|}{$85(19.36 \%)$} & \multicolumn{4}{|c|}{$90(20.50 \%)$} \\
\hline
\end{tabular}


Table 4. Comparison of Dyslipidemia Rates across Different Age Groups from 2017 to 2019 (\%).

\begin{tabular}{|c|c|c|c|c|c|c|c|c|c|c|c|c|}
\hline \multirow{2}{*}{ Age } & \multicolumn{3}{|c|}{$\mathrm{TC}$} & \multicolumn{3}{|c|}{ TG } & \multicolumn{3}{|c|}{ LDL-C } & \multicolumn{3}{|c|}{ HDL-C } \\
\hline & 2017 & 2018 & 2019 & 2017 & 2018 & 2019 & 2017 & 2018 & 2019 & 2017 & 2018 & 2019 \\
\hline $25 \sim 34$ & $3(6 \%)$ & $4(8 \%)$ & $6(12 \%)$ & 0 & $1(2 \%)$ & $3(6 \%)$ & $1(2 \%)$ & 0 & $1(2 \%)$ & 0 & 0 & 0 \\
\hline $35 \sim 44$ & $10(10.2 \%)$ & $8(8.16 \%)$ & $12(12.24 \%)$ & $7(7.14 \%)$ & $12(12.24 \%)$ & $14(14.29 \%)$ & $2(2.04 \%)$ & $1(1.02 \%)$ & 0 & $1(1.02 \%)$ & $5(5.10 \%)$ & $5(5.10 \%)$ \\
\hline $45 \sim 54$ & $12(8.16 \%)$ & $15(10.2 \%)$ & $14(9.52 \%)$ & $20(13.61 \%)$ & $25(17.0 \%)$ & $26(17.68 \%)$ & $1(0.68 \%)$ & $1(0.68 \%)$ & 0 & $2(1.36 \%)$ & $8(5.44 \%)$ & $16(10.88 \%)$ \\
\hline $55 \sim 65$ & $17(11.81 \%)$ & $19(13.19 \%)$ & $20(13.89 \%)$ & $24(16.67 \%)$ & $31(21.52 \%)$ & $25(17.36 \%)$ & $1(0.69 \%)$ & $2(1.39 \%)$ & 0 & $10(6.94 \%)$ & $27(18.75 \%)$ & $18(12.50 \%)$ \\
\hline Total & $42(9.57 \%)$ & $46(10.48 \%)$ & $52(11.85 \%)$ & $51(11.62 \%)$ & $69(15.72 \%)$ & $68(15.49 \%)$ & $5(1.14 \%)$ & $4(0.91 \%)$ & $1(0.23 \%)$ & $13(2.96 \%)$ & $40(9.11 \%)$ & $39(8.88 \%)$ \\
\hline
\end{tabular}




\section{References}

[1] Ren L, Bai L, Wu Y, et al. Prevalence of and risk factors for cognitive impairment among elderly without cardio-and cerebrovascular diseases: a population-based study in rural China. Front Aging Neuroscience, 2018, 10: 62.

[2] Shrivastava U, Misra A, Mohan V, et al. Obesity, diabetes and cardiovascular diseases in India: public health challenges. Curr Diabetes Rev, 2017, 13(1): 65-80.

[3] Shao P, Xu Y, Pan C W. Factors associated with and prevalence of depressive features amongst older adults in an urban city in eastern China. South African J Psy, 2017, 23(1).

[4] Akinyemi R O, Owolabi M O, Ihara M, et al. Stroke, cerebrovascular diseases and vascular cognitive impairment in Africa. Brain Res Bull, 2019, 145: 97-108.

[5] Schofield J D, Liu Y, Rao-Balakrishna P, et al. Diabetes dyslipidemia. Diabetes Therapy, 2016, 7(2): 203-219.

[6] Kopin L, Lowenstein C J. Dyslipidemia. Annals Int Med, 2017, 167(11): ITC81-ITC96.

[7] Ji Y, Bai C. Research progress of hypertriglyceridemia and coronary heart disease. Heart and Mind, 2018, 2(2): 40.

[8] Wang Y, Shen L, Xu D. Aerobic exercise reduces triglycerides by targeting apolipoprotein C3 in patients with coronary heart disease. Clinical Cardiology, 2019, 42(1): 56-61.

[9] Ohishi M. Hypertension with diabetes mellitus: physiology and pathology. Hypertension Research, 2018, 41(6): 389-393.

[10] Jellinger P S, Handelsman Y, Rosenblit P D, et al. American Association of Clinical Endocrinologists and American College of Endocrinology guidelines for management of dyslipidemia and prevention of cardiovascular disease. Endocrine Practice, 2017, 23(s2): 1-87.

[11] He N, Ye H. Exercise and Hyperlipidemia. Physical Exercise for Human Health. Springer, Singapore, 2020: 79-90.

[12] Albany C J, Trevelin S C, Giganti G, et al. Getting to the Heart of the Matter: The role of Regulatory T-cells (Tregs) in Cardiovascular Disease (CVD) and Atherosclerosis. Front Immunol, 2019, 10: 2795.

[13] Yang B Y, Guo Y, Markevych I, et al. Association of long-term exposure to ambient air pollutants with risk factors for cardiovascular disease in China. JAMA Network Open, 2019, 2(3): e190318-e190318.

[14] Zhou Z, Lu Y, Pi H, et al. Cadmium exposure is associated with the prevalence of 
dyslipidemia. Cell Phys Biochem, 2016, 40(3-4): 633-643.

[15] Wang Z, Li C, Yang Z, et al. Fetal and infant exposure to severe Chinese famine increases the risk of adult dyslipidemia: Results from the China health and retirement longitudinal study. BMC Public Health, 2017, 17(1): 488.

[16] de Assunção Bezerra M K, de Carvalho E F, Oliveira J S, et al. Health promotion initiatives at school related to overweight, insulin resistance, hypertension and dyslipidemia in adolescents: a cross-sectional study in Recife, Brazil. BMC Public Health, 2018, 18(1): 223. 
Figures
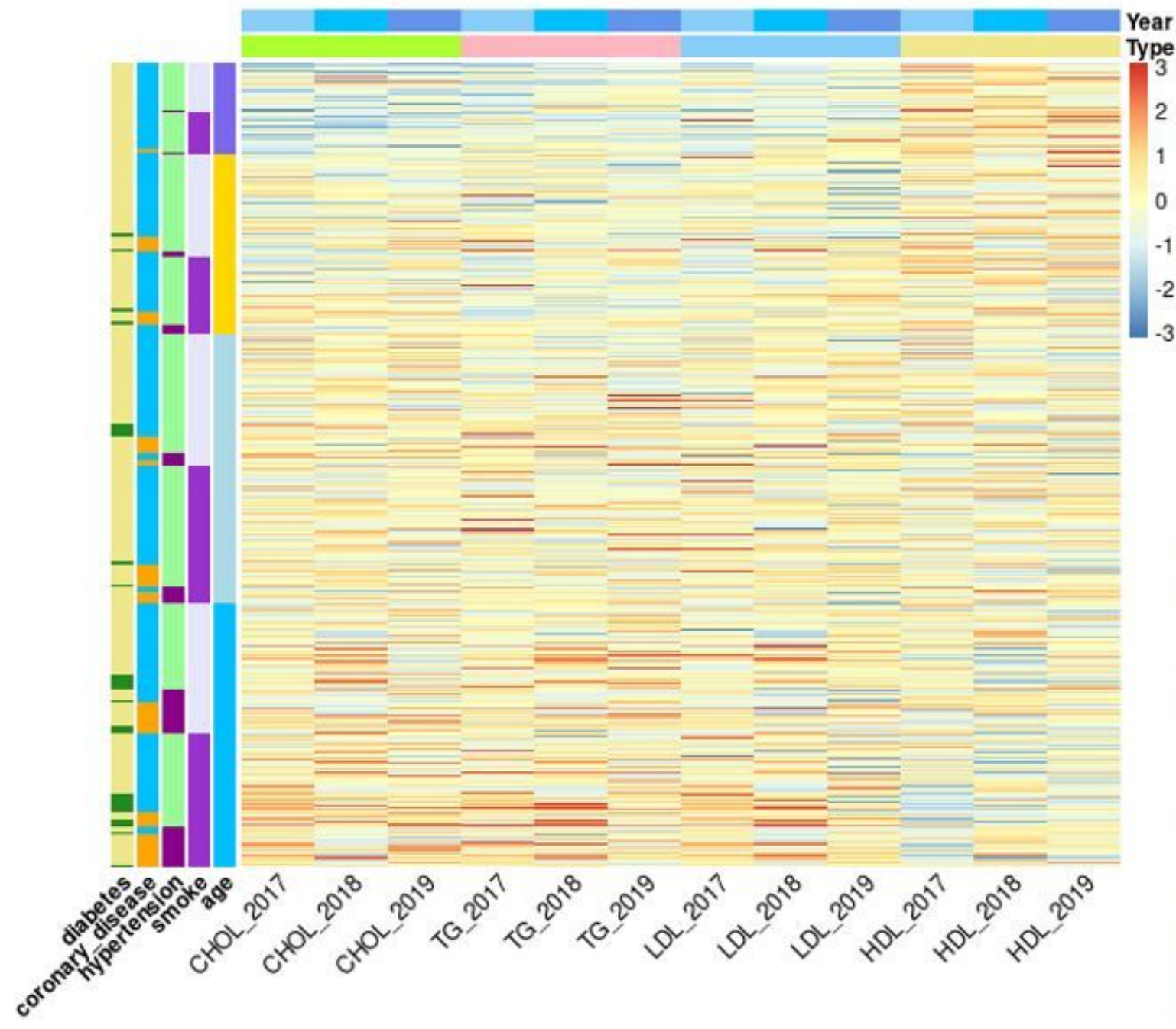

Type

3 Year

$2 \quad 2017$

2018

2019

0 Type

-1 CHOL

-2 TG

-3 LDL

HDL

diabetes

no
yes

coronary_disease

no
yes

hypertension

no
yes

smoke

no
yes

age

25 34

35 44

45 54

55 65

\section{Figure 1}

Analysis heatmap of risk factors related to plasma lipids (2017-2019). 